\title{
Design of an embedded sensor system for measuring laser scattering on blood cells
}

\author{
C. Iosifidis ${ }^{\mathrm{a}}$, K. Katsaliaki ${ }^{\mathrm{b}}$, P. Kollensperger ${ }^{\mathrm{c}}$ and M. E. Kiziroglou*a,d \\ ${ }^{a}$ Department of Automation Engineering T.E., ATEI Thessaloniki, 57400, Greece \\ ${ }^{\mathrm{b}}$ School of Economics, Business \& Legal Studies, International Hellenic University, 57001 Greece \\ ${ }^{c}$ Bionanotechnology Group, Norwegian University of Science and Technology, Trondheim, Norway \\ ${ }^{\mathrm{d}}$ Department of Electrical and Electronic Engineering, Imperial College London, SW7 2AZ, U.K.
}

\begin{abstract}
In this paper, a sensor system architecture for laboratory and in-vivo light scattering studies on blood cells is presented. It aims at correlating Mie scattering to compositional and physiological information of blood cells towards a non-invasive blood-cell counting sensor. An overview of previously reported experimental techniques on light scattering from blood cells is presented. State-of-the-art methods such as differential pulse measurements, vessel pressure optimization identified as promising for enhancing the scattering signal in such measurements. Indicative simulations of Mie scattering by blood cells are presented, illustrating the potential for distinguishing among cells and identifying size distribution. A prototype sensor system based on a 640-660 nm laser light source and a photo diode array is implemented and programmed to obtain mean amplitude and scattering angle measurements.
\end{abstract}

Keywords: Scattering, blood cells, non-invasive, sensor

\section{INTRODUCTION}

A technology that allows practical and non-invasive continuous monitoring of blood cells would have a profound major impact on the diagnosis and treatment of various pathological conditions and diseases. As an example, the pulse oximetry market is over $1 \mathrm{~b} \$$. Existing systems are able to monitor medical indicator quantities such as oxygenation [1], bilirubin [2] and hemoglobin [3] by spectrophotometric methods. A variety of techniques has also been proposed for non-invasive glucose monitoring [4]. Potential methods for non-invasive measurement of blood cells include spectrophotometry, light scattering, optical microscopy [5], acoustics and photo-acoustics [6].

In pulse oximetry, light transmission through a finger is monitored for a period of a few seconds, using two light emitting diodes. The emission of the one, typically at a low near infrared wavelength (e.g. $940 \mathrm{~nm}$ ), is more absorbed by oxygenated hemoglobin. The emission of the other, typically at a red wavelength (e.g. $660 \mathrm{~nm})$, is more absorbed by de-oxygenated hemoglobin. The percentage of oxygenation is determined by the relative transmission of the two wavelengths. In addition, from the variation of transmission through time, the pulse rate is determined. In order to eliminate the optical signal coming from non-blood regions of the finger, the instants of maximum and minimum transmission are detected. These correspond to maximum and minimum capillary volume. By subtracting the one transmission amplitude from the other, the non-blood related signal is rejected, achieving insensitivity to noise coming from conditions and specimen variations. A variety of commercial systems are available for home, athletics and medical use.

In optical hemoglobin monitoring systems, such as the OrSense NBM200, shown in Figure 1 (left), optical transmission measurements are used in combination with the application of controlled pressure on the finger, to maximize the desirable signal strength. This method has been coined with the name occlusion spectroscopy and it is patented by OrSense.

Another significant technique that is of interest to optical non-invasive monitoring of the blood composition and physiology is the signal-extraction algorithm for dropping venous signal resulting from body motion. This is also a proprietary algorithm and has been developed by Masimo Corporation. An example system is the Masimo rainbow oxymeter, which is able to monitor also hemoglobin, carboxyhemoglobin and methhemoglobin, using a combination of LEDs at seven different wavelengths.

*m.kiziroglou@autom.teithe.gr

Bio-MEMS and Medical Microdevices III, edited by Sander van den Driesche, Ioanna Gioroudi, Manuel Delgado-Restituto,Proc. of SPIE Vol. 10247, 102470G

(C) 2017 SPIE · CCC code: $0277-786 X / 17 / \$ 18 \cdot$ doi: 10.1117/12.2266515

Proc. of SPIE Vol. 10247 102470G-1 
Optical systems are also used for the measurement of bilirubin, especially for neo-natal screening. Such systems are based on reflection, rather than transmission measurements. In order achieve skin color and type invariance, modern bilirubinometers use a combination of a short and a long optical path, thereby distinguishing between reflections from the skin surface and from deeper skin. An example of such a system is the JM105 Draeger bilirubinometer (right of Fig. 1), which measures differentially at $450 \mathrm{~nm}$ and $550 \mathrm{~nm}$ [2]. In another technique for bilirubin determination, a microSpectrometer is employed. Such system measure and analyze reflection at 380-760 nm (tungsten halogen lamp). An example of such a system is the Philips BiliChek bilirubinometer [2]. A summary of the indicative non-invasive blood monitoring systems that have been discussed here is given in Table 1.
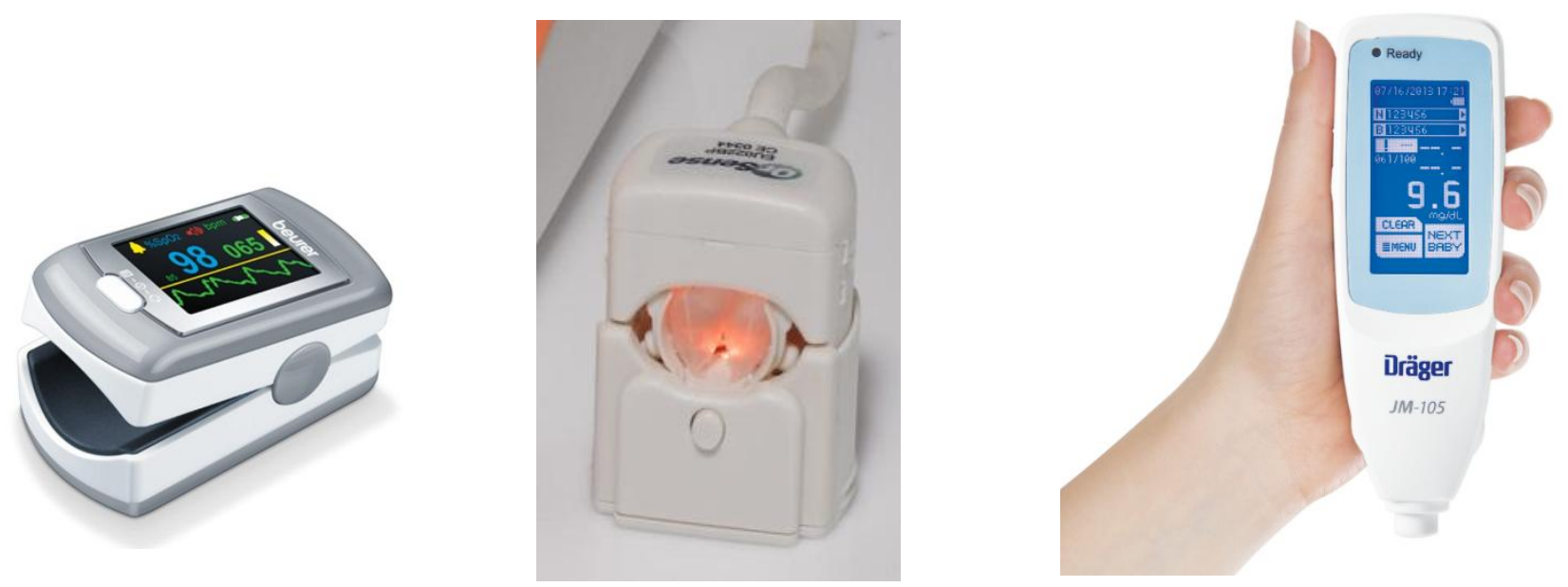

Fig. 1: Images of state-of-the-art commercially available non-invasive health monitoring systems. Left: The Beurer PO80 pulse oxymeter (image courtesy: Beurer Ltd). Middle:The OrSense NBM 200 Spectrolight sensor (image courtesy: OrSense Ltd). Right: The JM105 Draeger Bilirubin meter (image courtesy: Draeger AG).

\begin{tabular}{|c|c|c|}
\hline Model / Company & Quantities Measured & Methods \\
\hline $\begin{array}{l}\text { Rad-57 } \\
\text { Masimo, CA, USA }\end{array}$ & $\begin{array}{l}\mathrm{SpO}_{2} \text {, Respiration, } \\
\text { hemoglobin, } \\
\text { Meth/carboxy hemoglobin }\end{array}$ & $\begin{array}{l}\text { Differential Pulse } \\
\text { Differential wavelength } \\
\text { Seven different wavelengths } \\
\text { Body motion signal filtering }\end{array}$ \\
\hline $\begin{array}{l}\text { NBM } 200 \\
\text { OrSense, Israel }\end{array}$ & SpO2, hemoglobin & $\begin{array}{l}\text { Differential Pulse } \\
\text { Differential wavelength } \\
\text { Occlusion }\end{array}$ \\
\hline $\begin{array}{l}\text { JM103 } \\
\text { Konica Minolta, Japan }\end{array}$ & Bilirubin & $\begin{array}{l}\text { Different optical paths } \\
\text { Differential wavelength } \\
(450 \mathrm{~nm}-550 \mathrm{~nm})\end{array}$ \\
\hline $\begin{array}{l}\text { Bilichek } \\
\text { Philips, Netherlands }\end{array}$ & Bilirubin & $\begin{array}{l}\text { Different optical paths } \\
\text { Micro-spectroscopy } \\
(380-760 \mathrm{~nm})\end{array}$ \\
\hline
\end{tabular}

Table 1. Summary of some indicative, commercially available non-invasive blood monitoring systems. 
In the work presented in this paper, the possibility of using light scattering for the non-invasive determination of compositional and physiological information of blood cells is investigated. An overview of previously reported theoretical and experimental data on light scattering from blood cells is presented. Taking into account the state-of-the-art of existing optical non-invasive systems, outlined in the introduction, a new optical monitoring system is designed and fabricated. Laboratory experimental results are presented and analyzed. Finally, conclusions on system improvement and performance optimization factors are drawn and the necessary steps towards clinical testing are identified.

\section{LIGHT SCATTERING ON BLOOD CELLS}

A wealth of knowledge about light scattering from blood cells has become available in recent years [7-14]. Experimental studies typically involve the measurement of intensity as a function of scattering angle, and subsequent analysis using a suitable light scattering theory. For blood cells, the theory of light diffraction on spherical particles with diameter larger than the light wavelength, known as Mie scattering is usually employed. The light intensity vs angle pattern depends on the refractive index of the particle and the surrounding material as well as on the size of the particles. Therefore, it may be possible to estimate the size distribution of blood cells by analysis of their scattering pattern. An example of Mie scattering of different blood cells including lymphocytes, erythrocytes and other cells can be found in [10].

The intensity of Mie scattering is a wave - like function of angle, with an oscillating period and amplitude depending on the particle size and refractive index. As an indicative example, Mie scattering simulations have been performed and are presented in this paper, using the widely available diffraction software MiePlot [15], for red blood cells, white blood cells and platelets in surrounding plasma. The results are shown in Figure 2. The diameter and refractive index values used were $7 \mu \mathrm{m}$ and 1.38 for red blood cells [16], $13 \mu \mathrm{m}$ and 1.36 for white blood cells [17] and $2 \mu \mathrm{m}$ and 1.39 for platelets [18]. A plasma refractive index of 1.35 and a light wavelength of $650 \mathrm{~nm}$ were assumed [16].

The analysis of experimental light scattering data in comparison with theoretical expectations for single-size particles or for particles with a size range distribution may be assisted by performing a Fourier analysis of the intensity versus angle data. Such an approach has already been demonstrated to allow the discern between normal and pathological red blood cells [19].

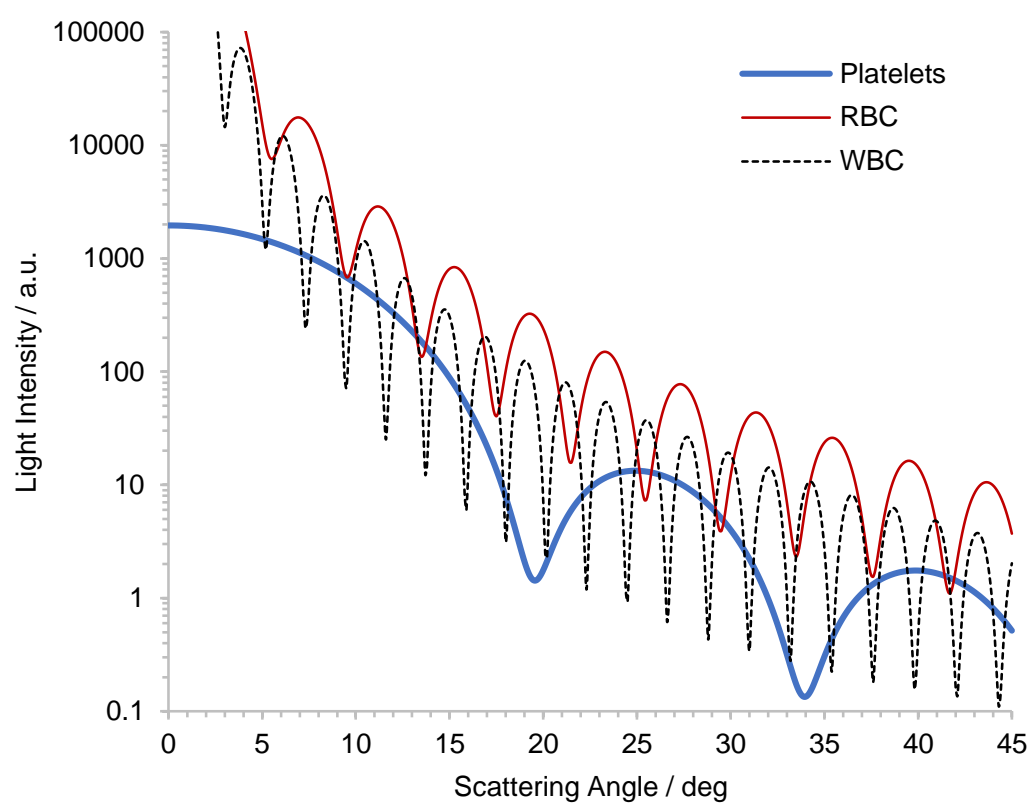

Figure 2: Mie scattering simulated using MiePlot [15], for red blood cells (RBC), white blood cells (WBC) and platelets in surrounding plasma. The diameter and refractive index values used were $7 \mu \mathrm{m}$ and 1.38 for RBC [16], $13 \mu \mathrm{m}$ and 1.36 for WBC [17] and $2 \mu \mathrm{m}$ and 1.39 for platelets [18]. 


\section{ARCHITECTURE OF THE PROPOSED SENSOR SYSTEM}

In order to study the effect of scattering on blood cells, a device architecture based on a directional light and a light sensor array is proposed. The main objective of this device design is to allow the detection, measurement and analysis of light scattering effects originating from blood cells, using as targets blood emulation suspension solutions, real blood samples but also the human finger, for in-vivo experiments. In the latter case, absorption and interface scattering must be taken into account. Accounting for a skin tissue absorption coefficient of $0.01 \mathrm{~mm}^{-1}$ [20], $10 \%$ of light power is absorbed while passing through a finger thickness of $10 \mathrm{~mm}$. The rest is scattered. In order to detect the outcoming scattered light, surrounding lighting, which is of the order of $0.5 \mu \mathrm{W} / \mathrm{mm}^{2}$ in typical indoor conditions [21], must be eliminated at the measurement point. The system should be able to consider, evaluate and possibly employ the state-of-the-art techniques summarized in the introduction of this paper. As discussed in the previous section, for data analysis and correlation to physical characteristics, the fitting of experimental results to Mie scattering models can be used [10, 19, 22]. This method can be combined with a study of the correlation of optical data with blood cell counts known from established invasive techniques such as complete blood counts (CBC). Such a clinical study could be performed in the frame of existing regular monitoring of patient groups with particular pathologies.

A schematic illustration of the device concept is presented in Figure 3. The system comprises a directional light source with a spot size in the range of $1 \mathrm{~mm}^{2}$ and a power of less than $1 \mathrm{~mW}$, such as a class 2 laser source. This power range was selected to simplify safety measures and minimize risks during device operation. The light beam passes through the specimen under study (e.g. a cuvette or the finger of a person) and the intensity of transmitted light at different scattering angles is detected using a photo diode array (PDA). Recordings of data are then captured for a duration and a sampling rate adequate for analysis over a few heart pulses.

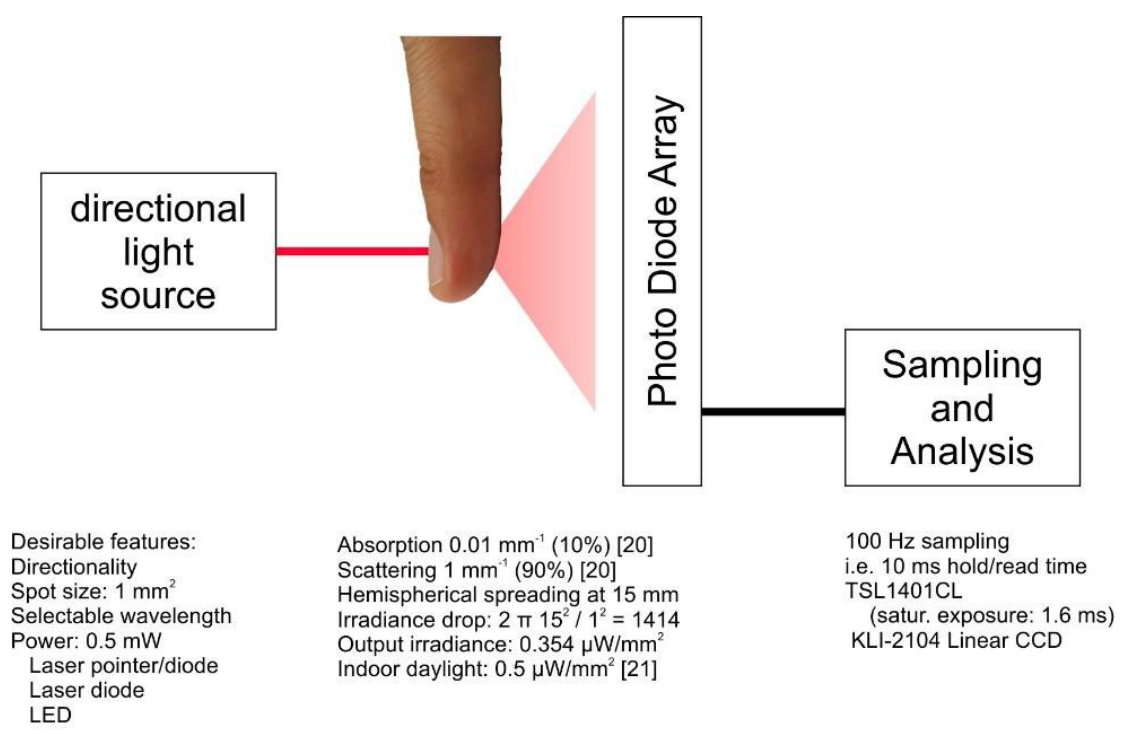

Figure 3: Schematic of the proposed system.

\section{DEVICE FABRICATION}

A prototype device has been fabricated, based on the architecture and approach presented in the previous section. A block diagram of the prototype system is shown in Figure 4. A class 2, Hama LP-15 red laser pointer has been used as the laser source, with an emission wavelength at $640-660 \mathrm{~nm}$, a power of $0.5 \mathrm{~mW}$ and a spot diameter of around $2 \mathrm{~mm}$. A $1 \mathrm{~mm}$ aperture is used to narrow the beam to a spot size that is more suitable for the detection of scattering angle by the given photodiode array. At a distance of $10 \mathrm{~mm}$ from the aperture, an AMS AG TSL 1401CL photodiode array is installed. This PDA contains an array of $128 \times 1$ photodiodes, with an active area length of $8 \mathrm{~mm}$. It has a controllable integration time (i.e. frame exposure time), allowing calibration to different specimens or optical setups for maximum sensitivity. The voltage output for a given irradiance and integration time (responsivity) of the device is $35 \mathrm{~V} /(\mu \mathrm{J} / \mathrm{cm} 2)$ at around $650 \mathrm{~nm}$, and the typical dark voltage is $0.1 \mathrm{~V}$. The output voltage range of the PDA is $0-4.8 \mathrm{~V}$ and the maximum clock frequency is $8 \mathrm{MHz}$. The full specifications of this device can be found in [23]. 
An Atmega 168-PU micro-controller unit (MCU) is employed for control and data acquisition, analysis and storage. This model includes an integrated 10-bit analogue to digital converter, programmable in a voltage range between 0-5 V, with a conversion time of $0.125 \mu \mathrm{s}$. A custom software is used to drive the PDA and collect data for a selectable duration, integration time and sample rate. Due to the low cache RAM of the MCU, each frame is stored to an external flash memory, connected through an SD interface. This SD interface is also used for transferring the acquired measurements to a computer for subsequent data analysis. Once the MCU is programmed, data scanning is triggered by an external button. An LED is used to indicate the completion of scanning activity.

\section{EXPERIMENTAL RESULTS}

The programming parameters of the fabricated prototype were calibrated by performing test scans and subsequent data analysis. For the specific optical setup used and in typical finger scanning measurements, an integration time of $50 \mathrm{~ms}$ was found to allow sufficient exposure for voltage output in the high end of the PDA output range, without leading to saturation. This corresponds to a frame rate of $20 \mathrm{~Hz}$, which is very low for electrocardiography (ECG) analysis [24], but adequate for capturing a heart rate pulse.

A test measurement on the finger of an adult was performed for a sampling period of five seconds. In Figure 5 left, the average detected irradiation across the PDA is plotted as a function of time. A clear detection of the expansion of capillaries, corresponding to the person's pulse is demonstrated. The corresponding pulse rate is $74 \pm 7 \%$ beats per minute. The measurement allows the observation of the morphology of the pulses. However, for an ECG analysis, a stronger signal and at a higher sampling rate would be desirable. Nevertheless, a normal 74 bps ECG calculated using the ECGSYN algorithm from [24] is also plotted as an indicative morphological reference.

A single frame of measured output voltage as a function of scattering angle is presented in Figure 5 right. The decay of irradiation amplitude with angle is detected, with a reduction of around $60 \%$ at 15 degrees. The oscillation observed has a period corresponding to the array pitch and it is a measurement artifact. A weaker oscillation with a period of around 3 degrees is also observed. Measurements of higher accuracy could allow the correlation of such oscillations to Mie scattering and the size, shape and population of blood cells, as discussed in section 2 of the paper.

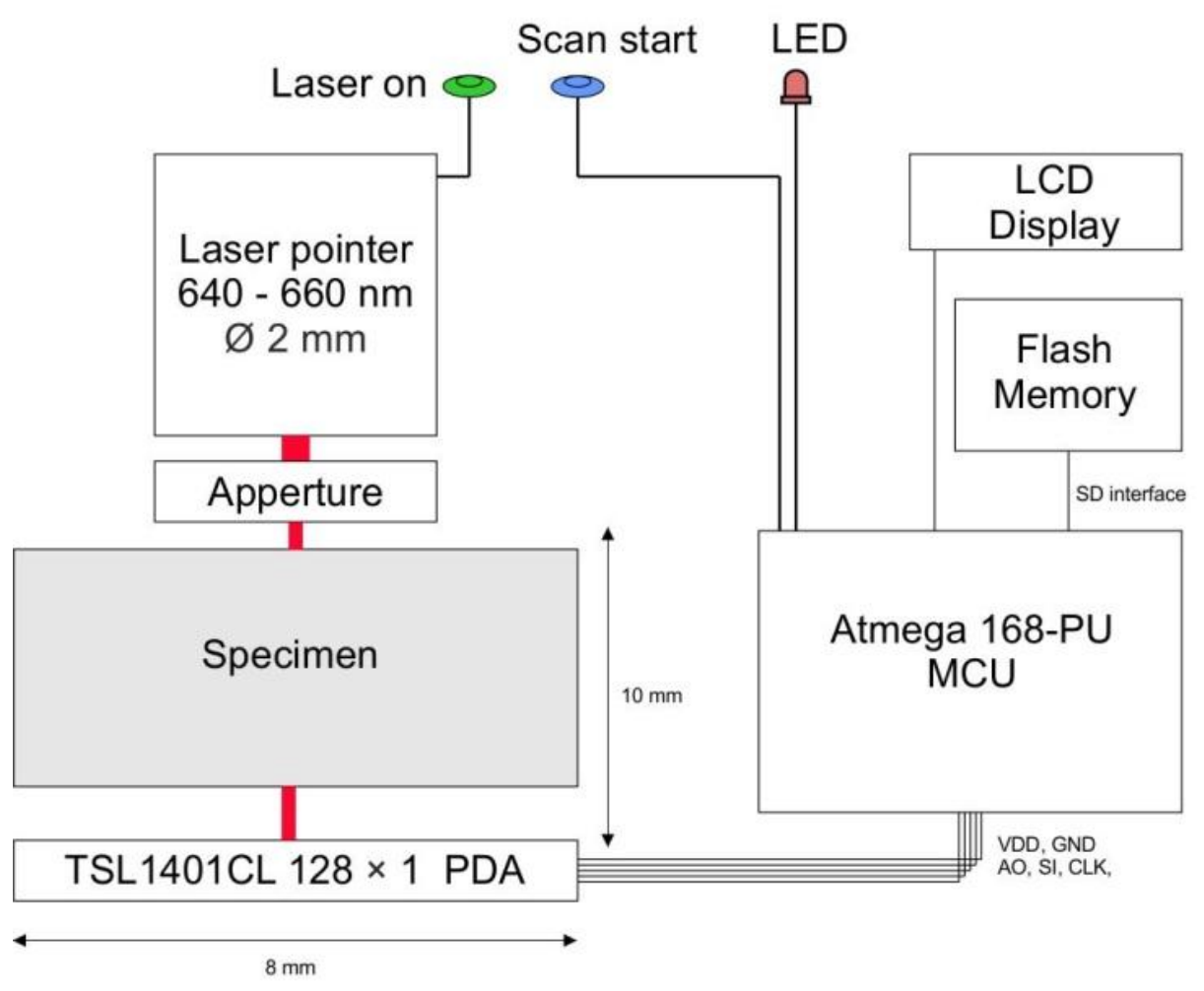

Figure 4: Block diagram of the prototype system 

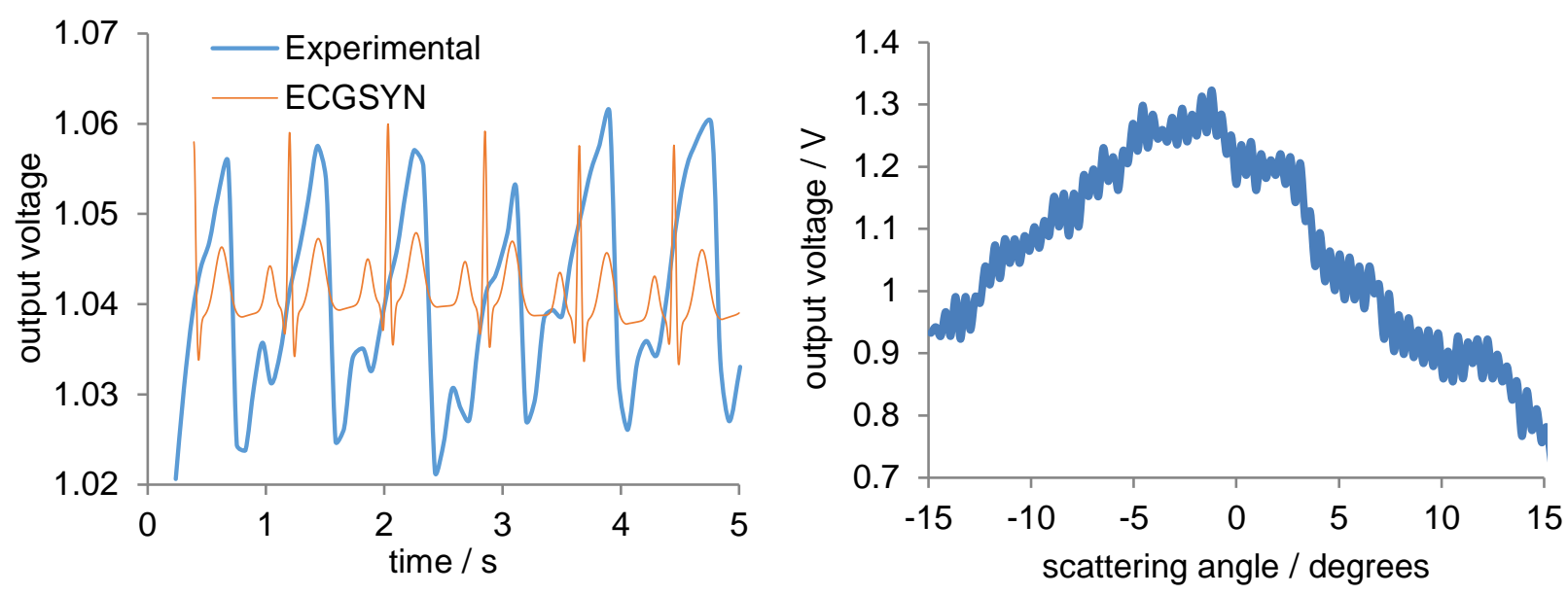

Figure 5: Left: Experimental measurements of average voltage across the photodiode array, corresponding to a $0.5 \mathrm{~mW}$ laser beam through the finger of an adult person. A heart rate of $74 \pm 7 \%$ beats per minute is extrapolated from the measurements. Simulated normal ECG data calculated using the ECGSYN algorithm [24] for a 74 bps pulse rate are also shown for comparison. Right: Experimental measurements of average voltage across the photodiode array, corresponding to a $0.5 \mathrm{~mW}$ laser beam through the finger of an adult person.

\section{CONCLUSIONS}

In this paper, a system architecture for studying laser scattering on blood cells was presented. The system is based on a $650 \mathrm{~nm}$ laser diode, a photo diode array and a microcontroller with data analysis and logging capability. It allows the implementation of key blood monitoring techniques such as differential pulse measurements, occlusion and noise filtering. The latter can be enhanced by integration of additional components such as motion, vibration or sound sensors. Comparison of scattering data with Mie theory predictions may allow distinction between particle sizes and types. Ongoing work involves the development of a compact implementation which can be evaluated using both reference latex sphere solutions and pre-measured biological samples. Subsequently, the system can be used for data collection from regularly tested populations in order to investigate the correlation between in-vivo collected light scattering data and blood cell size distribution and concentration.

\section{REFERENCES}

[1] J. M. Goldman, M. T. Petterson, R. J. Kopotic, and S. J. Barker, "Masimo signal extraction pulse oximetry," (in eng), J Clin Monit Comput, vol. 16, no. 7, pp. 475-83, 2000.

[2] S. N. El-Beshbishi, K. E. Shattuck, A. A. Mohammad, and J. R. Petersen, "Hyperbilirubinemia and Transcutaneous Bilirubinometry," Clinical Chemistry, vol. 55, no. 7, pp. 1280-1287, July 1, 20092009.

[3] I. Fine and A. Finarov, "Probe for use in non-invasive measurements of blood related parameters," ed: Google Patents, 2006.

[4] A. Tura, A. Maran, and G. Pacini, "Non-invasive glucose monitoring: Assessment of technologies and devices according to quantitative criteria," Diabetes Research and Clinical Practice, vol. 77, no. 1, pp. 16-40, 2007.

[5] T. Schwerte, D. Uberbacher, and B. Pelster, "Non-invasive imaging of blood cell concentration and blood distribution in zebrafish Danio rerio incubated in hypoxic conditions in vivo," (in eng), J Exp Biol, vol. 206, no. Pt 8, pp. 1299-307, Apr 2003.

[6] E. M. Strohm, E. S. Berndl, and M. C. Kolios, "Probing red blood cell morphology using high-frequency photoacoustics," (in eng), Biophys J, vol. 105, no. 1, pp. 59-67, Jul 22013.

[7] D. J. Faber, M. C. G. Aalders, E. G. Mik, B. A. Hooper, M. J. C. van Gemert, and T. G. van Leeuwen, "Oxygen Saturation-Dependent Absorption and Scattering of Blood," Physical Review Letters, vol. 93, no. 2, p. 028102, 2004.

[8] M. Friebel, A. Roggan, G. Müller, and M. Meinke, "Determination of optical properties of human blood in the spectral range 250to1100nm using Monte Carlo simulations with hematocrit-dependent effective scattering phase functions," Journal of Biomedical Optics, vol. 11, no. 3, pp. 034021-034021-10, 2006. 
[9] J. Li et al., "Noninvasive detection of functional brain activity with near-infrared diffusing-wave spectroscopy," Journal of Biomedical Optics, vol. 10, no. 4, pp. 044002-044002-12, 2005.

[10] V. P. Maltsev, "Scanning flow cytometry for individual particle analysis," Review of Scientific Instruments, vol. 71, no. 1, pp. 243-255, 2000.

[11] H. Martin, N. Y. Anna, and S. Dietrich, "A scattering phase function for blood with physiological haematocrit," Physics in Medicine and Biology, vol. 46, no. 3, p. N65, 2001.

[12] G. Popescu, "Chapter 5 Quantitative Phase Imaging of Nanoscale Cell Structure and Dynamics," in Methods in Cell Biology, vol. Volume 90, P. J. Bhanu, Ed.: Academic Press, 2008, pp. 87-115.

[13] G. Popescu, T. Ikeda, R. R. Dasari, and M. S. Feld, "Diffraction phase microscopy for quantifying cell structure and dynamics," Optics Letters, vol. 31, no. 6, pp. 775-777, 2006/03/15 2006.

[14] S. V. Tsinopoulos and D. Polyzos, "Scattering of He?Ne laser light by an average-sized red blood cell," Applied Optics, vol. 38, no. 25, pp. 5499-5510, 1999/09/01 1999.

[15] P. Laven, "MiePlot: a computer program for scattering of light from a sphere using Mie theory \& the Debye series, http://www.philiplaven.com/mieplot.htm," ed.

[16] Y. L. Jin, J. Y. Chen, L. Xu, and P. N. Wang, "Refractive index measurement for biomaterial samples by total internal reflection," Physics in Medicine and Biology, vol. 51, no. 20, p. N371, 2006.

[17] V. P. Maltsev, A. G. Hoekstra, and M. A. Yurkin, "Optics of White Blood Cells: Optical Models, Simulations, and Experiments," in Advanced Optical Flow Cytometry: Wiley-VCH Verlag GmbH \& Co. KGaA, 2011, pp. 63-93.

[18] I. V. Kolesnikova, S. V. Potapov, M. A. Yurkin, A. G. Hoekstra, V. P. Maltsev, and K. A. Semyanov, "Determination of volume, shape and refractive index of individual blood platelets," Journal of Quantitative Spectroscopy and Radiative Transfer, vol. 102, no. 1, pp. 37-45, 11// 2006.

[19] N. Ghosh, P. Buddhiwant, A. Uppal, S. K. Majumder, H. S. Patel, and P. K. Gupta, "Simultaneous determination of size and refractive index of red blood cells by light scattering measurements," Applied Physics Letters, vol. 88, no. 8, p. 084101, 2006.

[20] L. J. Steven, "Optical properties of biological tissues: a review," Physics in Medicine and Biology, vol. 58, no. 11, p. R37, 2013.

[21] M. Gorlatova, A. Wallwater, and G. Zussman, "Networking low-power energy harvesting devices: Measurements and algorithms," in 2011 Proceedings IEEE INFOCOM, 2011, pp. 1602-1610.

[22] K. B. Thovsen, "Evaluation of Mie scatter approximation formulas for the scattering of infrared light at biological cells," Master, Norwegian University of Life Sciences, 2013.

[23] "TSL1401CL $128 \times 1$ LINEAR SENSOR ARRAY WITH HOLD," 2011.

[24] P. E. McSharry, G. D. Clifford, L. Tarassenko, and L. A. Smith, "A dynamical model for generating synthetic electrocardiogram signals," (in eng), IEEE Trans Biomed Eng, vol. 50, no. 3, pp. 289-94, Mar 2003. 